\title{
OPEN IMAGES OF THE SORGENFREY LINE
}

\author{
VLAD SMOLIN
}

\begin{abstract}
We give a description of Hausdorff continuous open images of the Sorgenfrey line: these are precisely those spaces that have a Sorgenfrey base. Using this description we prove that no Hausdorff compact space that contains a copy of the Sorgenfrey line is a continuous open image of it; in particular the double-arrow space is not a continuous open image of the Sorgenfrey line.
\end{abstract}

Keywords: Sorgenfrey line, Souslin scheme, open map, double-arrow space, Hausdorff compact space

\section{INTRODUCTION}

A continuous map is called open if the image of an open set under this map is open.

Results in the paper arose from the questions that were posed to the author by E. G. Pytkeev and M. A. Patrakeev:

Question 1. Is the double-arrow (two-arrow) space a continuous open image of the Sorgenfrey line?

Question 2. Suppose that a compact space is a continuous open image of the Sorgenfrey line. What can we say about this space?

Previously, continuous open images of the Sorgenfrey line were studied in the class of metrizable spaces. In [7] S. A. Svetlichnyi proved that if a metrizable space is a continuous open image of the Sorgenfrey line, then it is a Polish space, i.e. separable completely metrizable space. In [5] and [6] N. V. Velichko and M. A. Patrakeev independently constructed a continuous open map from the Sorgenfrey line onto the real line. Velichko also proved that for each such map there exists a point with the preimage of cardinality continuum. In [3] Patrakeev proved that continuous open metrizable images of the Sorgenfrey line are exactly Polish spaces. He also strengthened the result of Velichko by showing that for each continuous open map from the Sorgenfrey line onto the metrizable space there exists a point with the preimage of cardinality continuum.

Continuous open images of submetrizable spaces were studied by Svetlichnyi. In 10. he proved that if a paracompact space is an image of a submetrizable space under continuous open compact map, then it is a submetrizable space. He also proved that there exists a nonmetrizable (hence not submetrizable) compact space that is a continuous open s-image of a submetrizable space. Since the Sorgenfrey line is a hereditarily separable submetrizable space it is natural to ask the following question.

Question 3. Is there exists a Hausdorff nonmetrizable compact space that is a continuous open image of the Sorgenfrey line? 
We give a description of Hausdorff open images of the Sorgenfrey line: these are precisely those spaces that have a Sorgenfrey base (see Theorem 1). Using this description we prove that no compact space that contains a homeomorphic copy of the Sorgenfrey line is an open image of it (see Corollary 4). This result answers Question 1 negatively. But the following question remains open.

Question 4. Is there exists a Hausdorff compact space that contains an uncountable subspace of the Sorgenfrey line and that is a continuous open image of the Sorgenfrey line?

\section{Notation AND TERMinOLOGY}

We use terminology from [1, 2], and [4]. Also we use the following notations:

Notation 1. The symbol $:=$ means "equals by definition"; the symbol $: \longleftrightarrow$ is used to show that the expression on the left side is an abbreviation for expression on the right side;

- $\omega:=$ the set of finite ordinals $=$ the set of natural numbers;

- $0=\emptyset \in \omega$;

- $n=\{0, \ldots, n-1\}$ for all $n \in \omega$;

- $s$ is a sequence $: \longleftrightarrow s$ is a function such that $\operatorname{dom}(s) \in \omega$ or $\operatorname{dom}(s)=\omega$;

- if $s$ is a sequence, then $\operatorname{lh}(s):=\operatorname{dom}(s)$;

- $\left\langle s_{0}, \ldots, s_{n-1}\right\rangle:=$ the sequence $s$ such that $\operatorname{lh}(s)=n \in \omega$ and $s(i)=s_{i}$ for all $i \in n$;

- \langle\rangle$:=$ the sequence $q$ such that $\operatorname{lh}(q)=0$;

- if $s=\left\langle s_{0}, \ldots, s_{n-1}\right\rangle$, then $s^{\wedge} x:=\left\langle s_{0}, \ldots, s_{n-1}, x\right\rangle$;

- $f\lceil A:=$ the restriction of function $f$ to $A$;

- if $s$ and $t$ are sequences, then $s \sqsubseteq t: \longleftrightarrow s=t \Gamma \operatorname{lh}(s)$;

- $s \sqsubset t: \longleftrightarrow s \sqsubseteq t$ and $s \neq t$;

- ${ }^{B} A:=$ the set of functions from $B$ to $A$;

- ${ }^{<\omega} A:=\bigcup_{n \in \omega}{ }^{n} A=$ the set of finite sequences in $A$.

Notation 2. Let $R$ be a binary relation on $X$ and $x, y \in X$. Then

- $x \uparrow_{R}:=\{z \in X: x R z\}$

- $x \downarrow_{R}:=\{z \in X: z R x\}$;

- $(x, y)_{R}:=x \uparrow_{R} \cap y \downarrow_{R}$.

Now we introduce several relations on ${ }^{<\omega} \omega$ and ${ }^{\omega} \omega$.

Notation 3. Let $a, b \in{ }^{<\omega} \omega \cup^{\omega} \omega$. Then

- $a \triangleleft b: \longleftrightarrow \exists n \in \omega$ such that

$-a\lceil n=b\lceil n \quad$ and

$-a(n)<b(n)$.

- $a \unlhd b: \longleftrightarrow a \triangleleft b$ or $a=b$.

Notation 4. Let $\langle X, \tau\rangle$ be a topological space, $x \in X, B \subseteq X$, and $A \subseteq \mathbb{R}$. Then

- $\omega^{\omega}:=$ the Baire space of weight $\aleph_{0}:=$ the countable power of the discrete space of cardinality $\aleph_{0}$;

- the double-arrow (two-arrow) space $:=\left\langle M, \tau_{\mathbb{A}}\right\rangle$, where $M=\{\langle x, 0\rangle: 0<$ $x \leq 1\} \cup\{\langle x, 1\rangle: 0 \leq x<1\}$ and $\tau_{\mathbb{A}}$ is the order topology induced by the lexicographic order on $M$ [2, b-13 Special Spaces]; 
- $\mathbb{S}:=$ the Sorgenfrey line $:=\left\langle\mathbb{R}, \tau_{\mathbb{S}}\right\rangle$, where $\tau_{\mathbb{S}}$ is the topology generated by $\{[a, b): a, b \in \mathbb{R}\}$

- $A_{\mathbb{S}}:=$ the set $A$ as a subspace of $\mathbb{S}$;

- $A_{\mathbb{R}}:=$ the set $A$ as a subspace of $\left\langle\mathbb{R}, \tau_{\mathbb{R}}\right\rangle$, where $\tau_{\mathbb{R}}$ is the natural topology on the real line;

- if $y \in \mathbb{R}$, then $y<A: \longleftrightarrow y<z$ for all $z \in A$;

- if $p \in{ }^{\omega} X$, then $p \stackrel{\langle X, \tau\rangle}{\longrightarrow} x: \longleftrightarrow p$ converges to $x$ in $\langle X, \tau\rangle$;

- $\operatorname{nbhds}(x, \tau):=\{U \in \tau: x \in U\}$

- $\tau\lceil B:=\{U \cap B: U \in \tau\}=$ the subspace topology of $B$;

- $\mathrm{Cl}_{\langle X, \tau\rangle}(B):=$ the closure of $B$ in $\langle X, \tau\rangle$;

- if $\langle Y, \sigma\rangle$ is a topological space, then $\langle X, \tau\rangle \cong\langle Y, \sigma\rangle: \longleftrightarrow\langle X, \tau\rangle$ is homeomorphic to $\langle Y, \sigma\rangle$.

Recall that [1] a Souslin scheme on a set $X$ is an indexed family $\mathbf{V}=\left\langle V_{a}\right\rangle_{a \in \epsilon^{<\omega} \omega}$ of subsets of $X$.

Definition 1. Let $\mathbf{V}=\left\langle V_{a}\right\rangle_{a \in<\omega_{\omega}}$ be a Souslin scheme on a set $X$ and $p \in{ }^{\omega} \omega$. Then

- $\operatorname{fruit}(\mathbf{V}, p):=\bigcap_{n \in \omega} V_{p \nmid n}$

- $\mathbf{V}$ is covering $: \longleftrightarrow V_{\langle\rangle}=X$ and $V_{a}=\bigcup_{n \in \omega} V_{a}{ }^{\wedge} n$ for all $a \in{ }^{<\omega} \omega$;

- $\mathbf{V}$ is complete $: \longleftrightarrow$ fruit $(\mathbf{V}, q) \neq \emptyset$ for all $q \in{ }^{\omega} \omega$;

- $\mathbf{V}$ has strict branches $: \longleftrightarrow|\operatorname{fruit}(\mathbf{V}, q)|=1$ for all $q \in{ }^{\omega} \omega$;

- $\mathbf{V}$ is locally strict $: \longleftrightarrow V_{a}=\bigcup_{n \in \omega} V_{a \wedge} n$ and $V_{a \wedge} m \cap V_{a \wedge k}=\emptyset$ for all $a \in{ }^{<\omega} \omega$ and $m \neq k \in \omega$.

Notation 5. Let $\mathbf{V}=\left\langle V_{a}\right\rangle_{a \in{ }^{<\omega} \omega}$ be a Souslin scheme on a set $X, x \in X, n \in \omega$, and $q \in{ }^{\omega} \omega$. Then

- $q$ is a branch of $x$ in $\mathbf{V}: \longleftrightarrow x \in$ fruit $(\mathbf{V}, q)$;

- branches $(\mathbf{V}, x):=$ the set of branches of $x$ in $\mathbf{V}$;

- rsubtree $(q, n):=\left\{s \in\left(q\lceil n) \uparrow_{\sqsubset} \cap<\omega \omega: q \triangleleft s\right\}\right.$;

- rsequences $(q, n):=\left\{p \in \omega^{\omega} \omega: q \triangleleft p \wedge q\lceil n=p\lceil n\}\right.$;

- $\operatorname{cut}(\mathbf{V}, q, n):=\bigcup\{\operatorname{fruit}(\mathbf{V}, p): p \in \operatorname{rsequences}(q, n)\}$.

Remark 1. Let $\mathbf{V}=\left\langle V_{a}\right\rangle_{a \in<\omega \omega}$ be a covering Souslin scheme on a set $X$, let $n, m \in \omega, n \leq m$, and $q, p \in{ }^{\omega} \omega$. Then

(i) rsequences $(q, m) \subseteq$ rsequences $(q, n)$;

(ii) if $p \unlhd q$ and $p\lceil n=q\lceil n$, then $\operatorname{rsequences}(q, n) \subseteq \operatorname{rsequences}(p, n)$;

(iii) $p \in \operatorname{rsequences}(q, n)$ iff $\exists k>n$ such that $p\lceil k \in \operatorname{rsubtree}(q, n)$;

(iv) $\operatorname{cut}(\mathbf{V}, q, n)=\bigcup\left\{V_{a}: a \in \operatorname{rsubtree}(q, n)\right\}=$ $=\left\{y \in V_{q \uparrow n}:\right.$ branches $\left.(\mathbf{V}, y) \cap \operatorname{rsequences}(q, n) \neq \emptyset\right\}$.

Definition 2. Let $\mathbf{V}=\left\langle V_{a}\right\rangle_{a \in<\omega}$ be a Souslin scheme on a set $X, \tau$ a topology on $X, x \in X$, and $q$ is a branch of $x$ in $\mathbf{V}$. Then

- $q$ is a $\tau$-base branch of $x$ in $\mathbf{V}: \longleftrightarrow\{\operatorname{cut}(\mathbf{V}, q, m) \cup\{x\}: m \in \omega\}$ is an open neighborhood base at the point $x$ in the space $\langle X, \tau\rangle$.

Because of this definition it is natural to introduce the following notation.

Notation 6. Let $\mathbf{V}=\left\langle V_{a}\right\rangle_{a \in \in^{<\omega} \omega}$ be a Souslin scheme on a set $X, x \in X$, and $q \in{ }^{\omega} \omega$. Then cutBase $(\mathbf{V}, q, x):=\{\operatorname{cut}(\mathbf{V}, q, m) \cup\{x\}: m \in \omega\}$. 
Remark 2. Let $\mathbf{V}=\left\langle V_{a}\right\rangle_{a \in \omega_{\omega} \omega}$ be a Souslin scheme on $X$ and $\tau$ a Hausdorff topology on $X$. Then for any sequence $q \in{ }^{\omega} \omega$ there is at most one point $x \in X$ such that $q$ is a $\tau$-base branch of $x$ in $\mathbf{V}$.

Notation 7. Let $\mathbf{V}=\left\langle V_{a}\right\rangle_{a \in<\omega}$ be a Souslin scheme on a set $X, \tau$ a topology on $X, x \in X$. Then

- $\mathrm{BB}(\mathbf{V}, x, \tau):=$ the family of $\tau$-base branches of $x$ in $\mathbf{V}$;

- Let $q \in{ }^{\omega} \omega$ is a $\tau$-base branch of some point $y$ in $\mathbf{V}$. Then using Remark 2 we define $\operatorname{pnt}(\mathbf{V}, q,\langle X, \tau\rangle):=$ the point $y \in X$ such that $q$ is a $\tau$-base branch of $y$ in $\mathbf{V}$.

\section{Notation 8.}

- $\mathbf{S}:=$ the Souslin scheme $\left\langle S_{a}\right\rangle_{a \in<\omega}{ }$ on ${ }^{\omega} \omega$ such that $S_{a}:=\left\{p \in{ }^{\omega} \omega: a \sqsubseteq p\right\}$ for all $a \in{ }^{<\omega} \omega$.

Remark 3. branches $(\mathbf{S}, p)=\{p\}$ for all $p \in{ }^{\omega} \omega$.

Let $\langle X, \tau\rangle$ be a topological space. Then a Souslin scheme $\mathbf{V}=\left\langle V_{a}\right\rangle_{a \in \epsilon^{<\omega} \omega}$ on the set $X$ is called an open Souslin scheme on the space $\langle X, \tau\rangle$ if $V_{a} \in \tau$ for all $a \in{ }^{<\omega} \omega$.

Definition 3. Let $\langle X, \tau\rangle$ be a Hausdorff topological space. Then an open complete covering Souslin scheme $\mathbf{V}=\left\langle V_{a}\right\rangle_{a \in \epsilon^{<\omega} \omega}$ on the space $\langle X, \tau\rangle$ is called a Sorgenfrey base for $\langle X, \tau\rangle$ if the following conditions hold:

(S1) $\forall x \in X \forall q \in$ branches $(\mathbf{V}, x) \forall n \in \omega$

$\exists t \in \mathrm{BB}(\mathbf{V}, x, \tau)(t\lceil n=q\lceil n)$;

(S2) $\forall q \in{ }^{\omega} \omega \exists z \in X(q \in \mathrm{BB}(\mathbf{V}, z, \tau))$.

Remark 4. Let $\mathbf{V}=\left\langle V_{a}\right\rangle_{a \in \omega_{\omega}}$ be a Sorgenfrey base for a Hausdorff topological space $\langle X, \tau\rangle$. Then branches $(\mathbf{V}, x) \subseteq \mathrm{Cl}_{\omega^{\omega}}(\mathrm{BB}(\mathbf{V}, x, \tau))$ for all $x \in X$.

\section{Description of open images of the Sorgenfrey line}

The main result of this section is the following theorem.

Theorem 1. A Hausdorff space is a continuous open image of the Sorgenfrey line iff there exists a Sorgenfrey base for this space.

Proof. The theorem follows from Corollary 1, Lemma 2, and Lemma 3 .

Lemma 1. The family $\bigcup\left\{\right.$ cutBase $\left.(\mathbf{S}, p, p): p \in{ }^{\omega} \omega\right\}$ is a base for a topology on ${ }^{\omega} \omega$ and for any point $x \in{ }^{\omega} \omega$ the family cutBase $(\mathbf{S}, x, x)$ is a neighborhood base at the point $x$ in this topology.

Proof. Let $p, q \in{ }^{\omega} \omega$, let $n, m \in \omega$, and let $x \in(\operatorname{cut}(\mathbf{S}, p, n) \cup\{p\}) \cap(\operatorname{cut}(\mathbf{S}, q, m) \cup$ $\{q\})$. We now prove that there exists a set $U \in \operatorname{cutBase}(\mathbf{S}, x, x)$ such that

$$
x \in U \subseteq(\operatorname{cut}(\mathbf{S}, p, n) \cup\{p\}) \cap(\operatorname{cut}(\mathbf{S}, q, m) \cup\{q\}) .
$$

Without loss of generality, we can assume that $n \leq m$. Consider the set $U:=$ $\operatorname{cut}(\mathbf{S}, x, m) \cup\{x\}$. Let $z \in U \backslash\{x\}$. Then using (i), (ii) of Remark11and Remark 3, we get $z \in \operatorname{rsequences}(x, m) \subseteq \operatorname{rsequences}(p, n) \cap \operatorname{rsequences}(q, m)$. Finally, from (i), (ii) of Remark 1 and Remark 3 it follows that $z \in(\operatorname{cut}(\mathbf{S}, p, n) \cup\{p\}) \cap(\operatorname{cut}(\mathbf{S}, q, m) \cup$ $\{q\})$.

Notation 9. $\sigma_{\mathbb{S}}:=$ the topology on ${ }^{\omega} \omega$ that is constructed in Lemma 1 . 
Remark 5. $\mathbf{S}$ is an open complete covering Souslin scheme on the space $\left\langle{ }^{\omega} \omega, \sigma_{\mathbb{S}}\right\rangle$.

Lemma 2. Let $\langle Y, \tau\rangle$ be a Hausdorff space and let $f:\left\langle{ }^{\omega} \omega, \sigma_{\mathbb{S}}\right\rangle \rightarrow\langle Y, \tau\rangle$ be an open continuous surjection. Denote $f\left[S_{a}\right]$ by $V_{a}$ for all $a \in{ }^{<\omega} \omega$. Then $\mathbf{V}:=\left\langle V_{a}\right\rangle_{a \in<\omega} \omega$ is a Sorgenfrey base for $\langle Y, \tau\rangle$.

Proof. Since $f$ is an open surjection and $\mathbf{S}$ is an open complete covering Souslin scheme, we see that $\mathbf{V}$ is an open complete covering Souslin scheme on the space $\langle Y, \tau\rangle$.

Let us prove that

$$
x \in \operatorname{branches}(\mathbf{V}, y) \quad \text { for all } y \in Y \text { and } x \in f^{-1}(y) .
$$

Let $y \in Y$ and $x \in f^{-1}(y)$. Then for any $n \in \omega$ it follows that $y=f(x) \in$ $f\left[S_{x\lceil n}\right]=V_{x\lceil n}$, and hence $x \in$ branches $(\mathbf{V}, y)$.

We now prove that

$$
x \in \mathrm{BB}(\mathbf{V}, y, \tau) \quad \text { for all } y \in Y \text { and } x \in f^{-1}(y) .
$$

Let $y \in Y$ and $x \in f^{-1}(y)$. Since cutBase $(\mathbf{S}, x, x)$ is an open neighborhood base at the poin $x$ and $f$ is an open map, it is enough to prove that $\operatorname{cut}(\mathbf{V}, x, n) \cup\{y\}=$ $f[\operatorname{cut}(\mathbf{S}, x, n) \cup\{x\}]$ for all $n \in \omega$.

Fix $n \in \omega$. Using (iv) of Remark 1, we get

$$
\begin{aligned}
& f[\operatorname{cut}(\mathbf{S}, x, n) \cup\{x\}]= \\
& f\left[\bigcup\left\{S_{a}: a \in \operatorname{rsubtree}(x, n)\right\}\right] \cup\{y\}= \\
& \bigcup\left\{f\left[S_{a}\right]: a \in \operatorname{rsubtree}(x, n)\right\} \cup\{y\}= \\
& \bigcup\left\{V_{a}: a \in \operatorname{rsubtree}(x, n)\right\} \cup\{y\}= \\
& \operatorname{cut}(\mathbf{V}, x, n) \cup\{y\} .
\end{aligned}
$$

Since $f$ is a surjection, it follows from (3) that $\mathbf{V}$ satisfies (S2).

We now prove that $\mathbf{V}$ satisfies (S1). Let $y \in Y, q \in \operatorname{branches}(\mathbf{V}, y)$, and $n \in \omega$. Then $y \in V_{q\lceil n}=f\left[S_{q\lceil n}\right]$. This means that there exists $x \in S_{q\lceil n}$ such that $f(x)=y$, so it follows from (3) that $x \in \mathrm{BB}(\mathbf{V}, y, \tau)$. Also since $x \in S_{q \mid n}$, we see that $x\lceil n=q\lceil n$.

Lemma 3. Let $\langle Y, \tau\rangle$ be a Hausdorff space with a Sorgenfrey base. Then there exists a continuous open surjection $f:\left\langle{ }^{\omega} \omega, \sigma_{\mathbb{S}}\right\rangle \rightarrow\langle Y, \tau\rangle$. Moreover, if $\langle Y, \tau\rangle$ has a locally strict Sorgenfrey base with strict branches, then $\langle Y, \tau\rangle$ is homeomorphic to $\left\langle{ }^{\omega} \omega, \sigma_{\mathbb{S}}\right\rangle$.

Proof. Let $\mathbf{V}=\left\langle V_{a}\right\rangle_{a \in \omega_{\omega}}$ be a Sorgenfrey base for $\langle Y, \tau\rangle$.

Let $f$ be the map from ${ }^{\omega} \omega$ to $Y$ such that $f(p):=\operatorname{pnt}(\mathbf{V}, p,\langle Y, \tau\rangle)$ for all $p \in$ ${ }^{\omega} \omega$. From property (S2) of $\mathbf{V}$ it follows that $f$ is a surjection. Let us prove that $f:\left\langle{ }^{\omega} \omega, \sigma_{\mathbb{S}}\right\rangle \rightarrow\langle Y, \tau\rangle$ is continuous and open.

Let $p \in{ }^{\omega} \omega$. Since $p \in \operatorname{BB}(\mathbf{V}, f(p), \tau)$, we see that cutBase $(\mathbf{V}, p, f(p))$ is an open neighborhood base at the point $f(p)$ in $\langle Y, \tau\rangle$. Hence we must only prove that

$$
f[\operatorname{cut}(\mathbf{S}, p, n) \cup\{p\}]=\operatorname{cut}(\mathbf{V}, p, n) \cup\{f(p)\} \text { for all } n \in \omega .
$$

Let $n \in \omega$. We will prove two inclusions. 
$" \subseteq "$ Let $q \in \operatorname{cut}(\mathbf{S}, p, n)$. From the definition of $f$ it follows that $q \in \operatorname{branches}(\mathbf{V}, f(q))$. From Remark 3 and (iv) of Remark 1 it follows that $q\lceil n=p\lceil n$ and $p \triangleleft q$. Finally, $f(q) \in \operatorname{cut}(\mathbf{V}, p, n)$.

" " Let $x \in \operatorname{cut}(\mathbf{V}, p, n)$. From (iv) of Remark 1 it follows that there exists $q \in \operatorname{branches}(\mathbf{V}, x)$ such that $q\lceil n=p\lceil n$ and $p \triangleleft q$. Choose $m \in \omega$ such that $q\lceil m=p\lceil m$ and $p\lceil(m+1) \triangleleft q \uparrow(m+1)$. From property (S1) of $\mathbf{V}$ it follows that there exists $t \in \mathrm{BB}(\mathbf{V}, x, \tau)$ such that $t \uparrow(m+1)=q \uparrow(m+1)$. Hence $t\lceil n=q\lceil n=p\lceil n$ and $p \triangleleft t$, so $t \in \operatorname{cut}(\mathbf{S}, p, n)$ by (iv) of Remark 1. Since $t \in \mathrm{BB}(\mathbf{V}, x, \tau)$, we see that $f(t)=x$.

Now suppose that $\mathbf{V}$ is locally strict and has strict branches. Then for any $x \in Y$ the set branches $(\mathbf{V}, x)$ is a singleton, therefore $f$ is a bijection because $f^{-1}(x) \subseteq \mathrm{BB}(\mathbf{V}, x, \tau) \subseteq \operatorname{branches}(\mathbf{V}, x)$.

Now we show that the Sorgenfrey line has a locally strict Sorgenfrey base with strict branches. To be precise, we show that a Lusin $\pi$-base for the Sorgenfrey line that was constructed in [3] has all this properties.

Example 1. We build a Souslin scheme $\mathbf{V}^{\mathbb{S}}=\left\langle V_{a}^{\mathbb{S}}\right\rangle_{a \in<\omega_{\omega}}$ by recursion on $\operatorname{lh}(a)$.

Let $\mathbf{V}_{\langle\rangle}^{\mathbb{S}}:=\mathbb{R}$, and let the set $\left\{\mathbf{V}_{a}^{\mathbb{S}}: \ln (a)=1\right\}$ equals $\{[i, i+1): i \in \mathbb{Z}\}$. When $\operatorname{lh}(a) \geq 1$, consider an interval $V_{a}^{\mathbb{S}}=[i, j)$. Let $\left\langle x_{n}\right\rangle_{n \in \omega}$ be a sequence in $\mathbb{R}$ such that $\left\langle x_{n}\right\rangle_{n \in \omega} \stackrel{\left\langle\mathbb{R}, \tau_{\mathbb{R}}\right\rangle}{\longrightarrow} j, x_{0}=i, x_{n+1}>x_{n}$, and $x_{n+1}-x_{n} \leq \frac{1}{\ln (a)+1}$. Define $V_{a \wedge n}^{\mathbb{S}}:=\left[x_{n}, x_{n+1}\right)$.

The next Corollary was first observed by Mikhail Patrakeev in private correspondence.

Corollary 1. $\mathbb{S} \cong\left\langle{ }^{\omega} \omega, \sigma_{\mathbb{S}}\right\rangle$.

Proof. The reader will easily prove that $\mathbf{V}^{\mathbb{S}}$ is a locally strict Sorgenfrey base for $\mathbb{S}$ that has strict branches. Then from Lemma 3 it follows that $\mathbb{S} \cong\left\langle{ }^{\omega} \omega, \sigma_{\mathbb{S}}\right\rangle$.

Corollary 2. The Sorgenfrey line is, up to homeomorphism, the unique Hausdorff topological space that has a locally strict Sorgenfrey base with strict branches.

\section{The spaces that are R-Bidirected along $\mathrm{Q}$}

Definition 4. Let $\langle X, \tau\rangle$ be a topological space, $Q$ a dense subset of $\langle X, \tau\rangle, R$ a binary relation on $X$, and $x \in X$. Then:

- An open neighborhood $U$ of $x$ is $R$-right along $Q$ if $x R y$ for all $y \in(U \backslash$ $\{x\}) \cap Q$

- we say that $x$ looks to the $R$-right along $Q$ if the following conditions hold:

- there exists an $R$-right along $Q$ open neighborhood of $x$;

- for any open neighborhood $U$ of $x$ there exists $y \in(U \backslash\{x\}) \cap Q$ such that $y \downarrow_{R}$ is a neighborhood of $x$.

- An open neighborhood $U$ of $x$ is $R$-left along $Q$ if $y R x$ for all $y \in(U \backslash$ $\{x\}) \cap Q$

- we say that $x$ looks to the $R$-left along $Q$ if the following conditions hold:

- there exists an $R$-left along $Q$ open neighborhood of $x$;

- for any open neighborhood $U$ of $x$ there exists $y \in(U \backslash\{x\}) \cap Q$ such that $y \uparrow_{R}$ is a neighborhood of $x$. 
Recall that a binary relation $R$ on a set $X$ is asymmetric if $x R y \Rightarrow \neg y R x$ for all $x, y \in X$.

Definition 5. Let $\langle X, \tau\rangle$ be a topological space, $Q$ a dense subset of $\langle X, \tau\rangle$, and $R$ an asymmetric binary relation on $X$. Then we say that $\langle X, \tau\rangle$ is $R$-bidirected along $Q$ if there are dense subsets $A_{l}, A_{r}$ of $\langle X, \tau\rangle$ such that

- $X=A_{l} \cup A_{r}$ and $A_{l} \cap A_{r}=\emptyset$;

- $x$ looks to the $R$-right along $\mathrm{Q}$ for all $x \in A_{r}$;

- $x$ looks to the $R$-left along $\mathrm{Q}$ for all $x \in A_{l}$.

Example 2. It is easy to check that the double-arrow space is $R$-bidirected along itself, where $R$ is the strict lexicographic order on it.

Lemma 4. Let $\langle X, \tau\rangle$ be a Hausdorff space. Suppose that there are a dense subset $Q$ of $\langle X, \tau\rangle$ and an asymmetric binary relation $R$ on $X$ such that $\langle X, \tau\rangle$ is $R$-bidirected along $Q$. Then $\langle X, \tau\rangle$ is not a continuous open image of $\mathbb{S}$.

Proof. Assume the converse. From Theorem 1 it follows that there exists $\mathbf{V}=$ $\left\langle V_{a}\right\rangle_{a \in<\omega \omega}$ is a Sorgenfrey base for $\langle X, \tau\rangle$. Let $A_{l}$ and $A_{r}$ be the sets from Definition 5. We build, by recursion on $n$, a sequence $\left\langle x_{n}\right\rangle_{n \in \omega}$ in $Q$, a sequence $\left\langle t_{n}\right\rangle_{n \in \omega}$ in ${ }^{\omega} \omega$, and an $\sqsubset$-increasing sequence $\left\langle p_{n}\right\rangle_{n \in \omega}$ in ${ }^{<\omega} \omega$ such that

(5) $\forall k \in \omega \exists x_{1}, x_{2} \in \operatorname{cut}(\mathbf{V}, p, k) \cap Q$ such that $x_{1} R z R x_{2}$ for all $z \in$ fruit $(\mathbf{V}, p)$,

where $p=\bigcup_{n \in \omega} p_{n}$.

Take $p_{0}:=\langle\rangle$. Suppose we have constructed $p_{0}, \ldots, p_{n} ; x_{0}, \ldots, x_{n-1} ;$ and $t_{0}, \ldots, t_{n-1}$. Now we consider two cases. Let $n$ be even. Then take any point $x \in V_{p_{n}} \cap A_{r}$. From property (S1) of $\mathbf{V}$ it follows that there exists $q \in \mathrm{BB}(\mathbf{V}, x, \tau)$ such that

$$
p_{n} \sqsubset q .
$$

Take $m>\operatorname{lh}\left(p_{n}\right)$ such that $\operatorname{cut}(\mathbf{V}, q, m) \cup\{x\} \subseteq V_{p_{n}}$. Since $x$ looks to the $R$-right along $Q$, we can take $x_{n} \in(\operatorname{cut}(\mathbf{V}, q, m) \backslash\{x\}) \cap Q$ such that

$$
x_{n} \downarrow_{R} \text { is a neighborhood of } x \text {. }
$$

From (iv) of Remark 1 it follows that we can take $t_{n} \in \operatorname{branches}\left(\mathbf{V}, x_{n}\right)$ such that

$$
t_{n} \in \operatorname{rsequences}(q, m) .
$$

Consider $k>m$ such that

$$
q\left\lceil k \triangleleft t_{n} \uparrow k .\right.
$$

Since $q \in \mathrm{BB}(\mathbf{V}, x, \tau)$, we see that there exists $a>k$ such that

$$
\operatorname{cut}(\mathbf{V}, q, a) \cup\{x\} \subseteq x_{n} \downarrow_{R} .
$$


Let $p_{n+1}$ be any element of rsubtree $(q, a)$. From (6) and inequalities $a>k>$ $m>\operatorname{lh}\left(p_{n}\right)$ it follows that $p_{n}=q\left\lceil\operatorname{lh}\left(p_{n}\right) \sqsubset q\left\lceil a \sqsubset p_{n+1}\right.\right.$. Since $p_{n+1} \in \operatorname{rsubtree}(q, a)$, from (iv) of Remark 1 and (10) it follows that

$$
z R x_{n} \text { for all } z \in V_{p_{n+1}} \text {. }
$$

Now we show that

$$
p_{n} \sqsubset t_{n} \text { and } p_{n+1} \triangleleft t_{n} \mid \operatorname{lh}\left(p_{n+1}\right) .
$$

From inequality $m>\ln \left(p_{n}\right)$, (6), and (8) it follows that $p_{n} \sqsubset t_{n}$. To prove the second part of (12), we must observe that $q\left\lceil k \sqsubset p_{n+1}\right.$ and use (9).

Now let $n$ be odd. If we argue as above by taking $x \in V_{p_{n}} \cap A_{l}, x_{n}$ such that $x_{n} \uparrow_{R}$ is a neighborhood of $x$, and $t_{n} \in \operatorname{branches}(\mathbf{V}, \times(n))$, then we can choose $p_{n+1}$ such that

$$
x_{n} R z \text { fol all } z \in V_{p_{n+1}}
$$

and

$$
p_{n} \sqsubset t_{n} \text { and } p_{n+1} \triangleleft t_{n} \mid \operatorname{lh}\left(p_{n+1}\right) .
$$

We now prove (5). Let $k \in \omega, n>k$, and $n$ is even. Then $p \nmid k \sqsubset p_{n}$. So from (12) it follows that $t_{n} \in \operatorname{rsequences}(p, k)$. Hence using (iv) of Remark 1, we get $x_{n} \in \operatorname{cut}(\mathbf{V}, p, k)$. Finally, from (11) it follows that $z R x_{n}$ for all $z \in \operatorname{fruit}(\mathbf{V}, p) \subseteq$ $V_{p_{n+1}}$. If we argue as above by taking $m>k$ such that $m$ is odd, then we can show that $x_{m} \in \operatorname{cut}(\mathbf{V}, p, k)$ and $x_{m} R z$ for all $z \in$ fruit $(\mathbf{V}, p) \subseteq V_{p_{m+1}}$.

Let us prove that

$$
\forall z \in \text { fruit }(\mathbf{V}, p): p \notin \mathrm{BB}(\mathbf{V}, z, \tau) .
$$

Let $z \in$ fruit $(\mathbf{V}, p)$, we consider two cases.

Case 1: $z \in A_{r}$. From (5) it follows that for any $k \in \omega$ there exists $x_{1} \in$ $\operatorname{cut}(\mathbf{V}, p, k) \cap Q$ such that $x_{1} R z$, and so $\neg z R x_{1}$ and $z \neq x_{1}$. Hence for all $k \in \omega$ if $\operatorname{cut}(\mathbf{V}, p, k) \cup\{z\}$ is an open neighborhood of $z$, then it is not $R$-right along $Q$. And since $z$ looks to the $R$-right along $Q$, we see that cutBase $(\mathbf{V}, p, z)$ is not an open neighborhood base at the point $z$. So $p \notin \mathrm{BB}(\mathbf{V}, z, \tau)$.

Case 2: $z \in A_{l}$. Arguing as above, we can take $x_{2} \in \operatorname{cut}(\mathbf{V}, p, k) \cap Q$ such that $z R x_{2}$, and so $p \notin \mathrm{BB}(\mathbf{V}, z, \tau)$.

Formula (15) contradicts property $(\mathrm{S} 2)$ of $\mathbf{V}$. The lemma is proved.

\section{The Spaces that are not open images of the Sorgenfrey line}

A subset of a topological space is called co-dense if its complement is dense.

Theorem 2. Suppose that $\langle X, \tau\rangle$ is a Hausdorff topological space, $S \subseteq X$ is a dense and co-dense subset of $X$. If $\langle S, \tau\lceil S\rangle$ is homeomorphic to the Sorgenfrey line, then $\langle X, \tau\rangle$ is not a continuous open image of the Sorgenfrey line. 
Proof. Since $[0,1)_{\mathbb{S}} \cong \mathbb{S}$, without loss of generality, we can assume that $S=[0,1)$ and $[0,1)_{\mathbb{S}}=\langle S, \tau\lceil S\rangle$.

If $\langle X, \tau\rangle$ is not first-countable, then it is not a continuous open image of the Sorgenfrey line, because the first axiom of countability is preserved by continuous open maps. So suppose that $\langle X, \tau\rangle$ is first-countable.

Using Lemma 4 it is enough to prove that there exists an asymmetric relation $R$ on $X$ such that $\langle X, \tau\rangle$ is $R$-bidirected along $[0,1)$. For each $A \subseteq X$, by $\breve{A}$ we denote $A \cap[0,1)$.

Denote by $\mathrm{L}$ the function with domain $X \backslash[0,1)$ such that for all $z \in X \backslash[0,1)$

$$
\mathrm{L}(z):=\left\{x \in[0,1]: \exists p \in{ }^{\omega}[0,1)\left(p \stackrel{\langle X, \tau\rangle}{\longrightarrow} z \wedge p \stackrel{[0,1]_{\mathbb{R}}}{\longrightarrow} x\right)\right\} .
$$

Since $\langle X, \tau\rangle$ is a first countable space and $[0,1)$ is dense in it, we see that $\mathrm{L}(z) \neq \emptyset$ for all $z \in X \backslash[0,1)$. We prove that

$$
<\text { well-orders } \mathrm{L}(z) \text { for all } z \in X \backslash[0,1) \text {. }
$$

Let $z \in X \backslash[0,1)$. Assume the converse. Let $q \in{ }^{\omega} \mathrm{L}(z)$ be such that $q(n+1)<$ $q(n)$ for all $n \in \omega$. Consider $x \in[0,1)$ such that

$$
q \stackrel{[0,1)_{\mathrm{S}}}{\longrightarrow} x
$$

Let us prove that

$$
U_{z} \cap U_{x} \neq \emptyset \text { for all } U_{x} \in \operatorname{nbhds}(x, \tau) \text { and } U_{z} \in \operatorname{nbhds}(z, \tau) \text {. }
$$

Let $U_{x} \in \operatorname{nbhds}(x, \tau)$ and $U_{z} \in \operatorname{nbhds}(z, \tau)$. Without loss of generality, we can assume that $\breve{U}_{x}=[x, x+\varepsilon)$. From (17) it follows that there exists $n \in \omega$ such that $q(n) \in \breve{U}_{x} \subseteq U_{x}$. Since $q(n) \in \mathrm{L}(z)$, there exists $p \in{ }^{\omega}[0,1)$ such that $p \stackrel{\langle X, \tau\rangle}{\longrightarrow} z$ and $p \stackrel{[0,1]_{\mathbb{R}}}{\longrightarrow} q(n)$. Since $q(n) \neq x$, we obtain $q(n) \in(x, x+\varepsilon)$, and so $p$ is eventually in $(x, x+\varepsilon) \subseteq U_{x}$. Also $p$ is eventually in $U_{z}$, hence $U_{x} \cap U_{z} \neq \emptyset$. Formula (18) contradicts the Hausdorff property of $\langle X, \tau\rangle$, so (16) is proved.

Denote by $\mathrm{m}$ the function that takes each point $z \in X \backslash[0,1)$ to the $<$-minimal element of $\mathrm{L}(z)$. Also let $\mathrm{M}$ be the function that takes each point $z \in X \backslash[0,1)$ to the $\sup _{[0,1]}(\mathrm{L}(z))$. Now we prove a technical lemma about this functions.

Lemma 5. Let $z \in X \backslash[0,1)$ and $a, b \in \mathbb{R}$ such that $a<b$. Then

(i) $\exists U \in \operatorname{nbhds}(z, \tau)(\breve{U}<\mathrm{M}(z))$;

(ii) $\forall U \in \operatorname{nbhds}(z, \tau)(\breve{U}=[a, b) \rightarrow \mathrm{M}(z) \leq b)$;

(iii) $\forall x \in[0,1)(x<\mathrm{m}(z) \rightarrow \exists U \in \operatorname{nbhds}(z, \tau)(x<\breve{U}))$;

(iv) $\forall x \in[0,1) \forall U \in \operatorname{nbhds}(z, \tau)(x<\breve{U} \rightarrow x<\mathrm{m}(z))$;

(v) $\forall U \in \operatorname{nbhds}(z, \tau) \exists x \in \breve{U}(x<\mathrm{m}(z))$.

Proof. (i). Assume the converse. Assume that for any $U \in \operatorname{nbhds}(z, \tau)$ there exists $x \in \breve{U}$ such that $\mathrm{M}(z) \leq x$. Let $p \in{ }^{\omega}[0,1)$ be such that

$$
\forall n \in \omega(\mathrm{M}(z) \leq p(n))
$$

and

$$
p \stackrel{\langle X, \tau\rangle}{\longrightarrow} z
$$


Take $x \in[0,1]$ such that $p^{\prime} \stackrel{[0,1]_{\mathbb{R}}}{\longrightarrow} x$, where $p^{\prime}$ is a subsequence of $p$. Then from (20) it follows that $p^{\prime} \stackrel{\langle X, \tau\rangle}{\longrightarrow} z$, and so $x \in \mathrm{L}(z)$. From (19) it follows that $\mathrm{M}(z) \leq x$. From equality $\mathrm{M}(z)=\sup _{[0,1]}(\mathrm{L}(z))$ it follows that $x=\mathrm{M}(z)$, and so using (19), we get $p^{\prime} \stackrel{[0,1)_{\mathbb{S}}}{\longrightarrow} \mathrm{M}(z)$. Hence $p^{\prime} \stackrel{\langle X, \tau\rangle}{\longrightarrow} \mathrm{M}(z)$. This contradicts the Hausdorff property of $\langle X, \tau\rangle$, so we prove (i).

(ii). Assume the converse. Suppose that there exists $U \in \operatorname{nbhds}(z, \tau)$ such that $\breve{U}=[a, b)$ and $b<\mathrm{M}(z)$. Since $\mathrm{M}(z)=\sup _{[0,1]}(\mathrm{L}(z))$, we see that there exists $q \in{ }^{\omega} \mathrm{L}(z)$ such that

$$
q \stackrel{[0,1]_{\mathbb{R}}}{\longrightarrow} \mathrm{M}(z)
$$

and

$$
q(n) \leq \mathrm{M}(z) \text { for all } n \in \omega .
$$

Hence there exists $m \in \omega$ such that $b<q(m) \leq \mathrm{M}(z) . q(m) \in \mathrm{L}(z)$, so there exists $p \in{ }^{\omega}[0,1)$ such that

$$
p \stackrel{\langle X, \tau\rangle}{\longrightarrow} z
$$

and

$$
p \stackrel{[0,1]_{\mathbb{R}}}{\longrightarrow} q(m) .
$$

Let $\varepsilon>0$ be such that $b<(q(m)-\varepsilon, q(m)+\varepsilon)$. Then $(q(m)-\varepsilon, q(m)+\varepsilon) \cap U=\emptyset$ and $p$ is eventually in $\left(x_{m}-\varepsilon, x_{m}+\varepsilon\right)$. This contradicts formula (21), so we prove (ii).

(iii). Assume the converse. Suppose that there exists $x \in[0,1)$ such that $x<$ $\mathrm{m}(z)$ and $\forall U \in \operatorname{nbhds}(z, \tau)$ there exists $y \in U$ such that $y \leq x$. Take $p \in{ }^{\omega}[0,1)$ such that

$$
p \stackrel{\langle X, \tau\rangle}{\longrightarrow} z
$$

and

$$
p(n) \leq x \text { for all } n \in \omega .
$$

Consider $y \in[0,1]$ such that $p^{\prime} \stackrel{[0,1]_{\mathbb{R}}}{\longrightarrow} y$, where $p^{\prime}$ is a subsequence of $p$. Then from (23) it follows that $p^{\prime} \stackrel{\langle X, \tau\rangle}{\longrightarrow} z$, and so $y \in \mathrm{L}(z)$. From (24) it follows that $y \leq x<\mathrm{m}(z)$, this contradicts $\mathrm{m}(z)$ is the $<$-minimal element of $\mathrm{L}(z)$, so we prove (iii).

(iv). Assume the converse. Suppose that there exists $x \in[0,1)$ and $U \in$ $\operatorname{nbhds}(z, \tau)$ such that $x<\breve{U}$ and $\mathrm{m}(z) \leq x$. Since $\mathrm{m}(z) \in \mathrm{L}(z)$, we see that there exists $p \in{ }^{\omega}[0,1)$ such that

$$
p \stackrel{\langle X, \tau\rangle}{\longrightarrow} z
$$

and

$$
p \stackrel{[0,1]_{\mathbb{R}}}{\longrightarrow} \mathrm{m}(z) .
$$

Now let us consider two cases. Case 1: $x=\mathrm{m}(z)$. Since $x<\breve{U}$, from (25) it follows that there exists $n \in \omega$ such that $x<p(k)$ for all $k>n$. Hence using (26) 
and equality $x=\mathrm{m}(z)$, we get $p \stackrel{[0,1)_{\mathbb{S}}}{\longrightarrow} x$, and so $p \stackrel{\langle X, \tau\rangle}{\longrightarrow} x$. This contradicts the Hausdorff property of $\langle X, \tau\rangle$.

Case 2: $x>\mathrm{m}(z)$. Take $\varepsilon>0$ such that $(\mathrm{m}(z)-\varepsilon, \mathrm{m}(z)+\varepsilon)<x$. Since $x<\breve{U}$, we see that $(\mathrm{m}(z)-\varepsilon, \mathrm{m}(z)+\varepsilon) \cap U=\emptyset$. From (26) it follows that $p$ is eventually in $(\mathrm{m}(z)-\varepsilon, \mathrm{m}(z)+\varepsilon)$, but this contradicts formula (25), so we prove (iv).

(v). Assume the converse. Suppose that there exists $U \in \operatorname{nbhds}(z, \tau)$ such that

$$
\mathrm{m}(z) \leq x \text { for all } x \in \breve{U} .
$$

From $\mathrm{m}(z) \in \mathrm{L}(z)$ it follows that there exists $p \in{ }^{\omega}[0,1)$ such that

$$
p \stackrel{\langle X, \tau\rangle}{\longrightarrow} z
$$

and

$$
p \stackrel{[0,1]_{\mathbb{R}}}{\longrightarrow} \mathrm{m}(z) .
$$

From (28) it follows that $p$ is eventually in $U$, and so from (27) and (29) it follows that $p \stackrel{[0,1)_{\mathbb{S}}}{\longrightarrow} \mathrm{m}(z)$, hence $p \stackrel{\langle X, \tau\rangle}{\longrightarrow} \mathrm{m}(z)$. This contradicts the Hausdorff property of $\langle X, \tau\rangle$, so we prove $(\mathrm{v})$.

Define

$$
\begin{aligned}
x R y \Longleftrightarrow x & <y \vee \\
& y \in X \backslash[0,1) \wedge x<\mathrm{M}(y) \vee \\
& x \in X \backslash[0,1) \wedge \mathrm{M}(x) \leq y .
\end{aligned}
$$

It is not hard to prove that $R$ is asymmetric. Define $A_{r}:=[0,1)$ and $A_{l}:=$ $X \backslash[0,1)$. Note that

$$
X=A_{r} \cup A_{l} \text { and } A_{r} \cap A_{l}=\emptyset .
$$

Let us prove that

$$
x \text { looks to the } R \text {-right along }[0,1) \text { for all } x \in A_{r} \text {. }
$$

Let $x \in A_{r}=[0,1)$. There exists $W \in \operatorname{nbhds}(x, \tau)$ such that $\breve{W}=[x, 1)$. It is easy to prove that $W$ is $R$-right along $[0,1)$.

Now let $U$ be an arbitrary element of $\operatorname{nbhds}(x, \tau)$. Take $y \in \breve{U}$ such that $x<y$ and $V \in \operatorname{nbhds}(x, \tau)$ such that $\breve{V}=[x, y)$. Let us prove that

$$
V \subseteq y \downarrow_{R}
$$

Let $z \in V$. If $z \in \breve{V}$, then $z<y$, and so $z R y$. Suppose that $z \in V \backslash[0,1)$. Then from (ii) of Lemma 5 it follows that $\mathrm{M}(z) \leq y$, and so by definition of $R$, we get $z R y$.

Now we shall prove that

$$
z \text { looks to the } R \text {-left along }[0,1) \text { for all } z \in A_{l} \text {. }
$$

Let $z \in A_{l}=X \backslash[0,1)$. From (i) of Lemma [5] it follows that there exists $U \in \operatorname{nbhds}(z, \tau)$ such that $\breve{U}<\mathrm{M}(z)$, and so by definition of $R$, we get $x R z$ for all $x \in \breve{U}$, hence $U$ is an $R$-left along $[0,1)$. 
Now let $U$ be an arbitrary element of $\operatorname{nbhds}(z, \tau)$. From (v) of Lemma 5 it follows that there exists $x \in \breve{U}$ such that

$$
x<\mathrm{m}(z) .
$$

From (33) and (iii) of Lemma [5 it follows that there exists $V \in \operatorname{nbhds}(z, \tau)$ such that

$$
x<\breve{V}
$$

Let us prove that

$$
V \subseteq x \uparrow_{R}
$$

Let $y \in V$. If $y \in \breve{V}$, then from (34) it follows that $x<y$, and so $x R y$. Let $y \in V \backslash[0,1)$. Then from (34) and (iv) of Lemma 5 it follows that $x<\mathrm{m}(y) \leq \mathrm{M}(y)$, and so by definition of $R$, we get $x R y$.

From (30), (31), and (32) it follows that $\langle X, \tau\rangle$ is $R$-bidirected along $[0,1)$.

Corollary 3. If $b \mathbb{S}$ is a Hausdorff compactification of the Sorgenfrey line, then it is not a continuous open image of the Sorgenfrey line.

Proof. By $b \mathbb{S}^{*}$ denote $b \mathbb{S} \backslash \mathbb{S}$. From [9, Ch. 4 , Pr. 43] it follows that $b \mathbb{S}^{*}$ is a dense subset of $b \mathbb{S}$. Then from Theorem 2 it follows that $b \mathbb{S}$ is not a continuous open image of the Sorgenfrey line.

Lemma 6. The property of being a continuous open image of the Sorgenfrey line is preserved by closed subspaces without isolated points.

Proof. Let $\langle X, \tau\rangle$ be a topological space such that there exists a continuous open surjection $f: \mathbb{S} \rightarrow\langle X, \tau\rangle$. Let $F \subseteq X$ be a closed subset of $\langle X, \tau\rangle$ without isolated points and $Z:=f^{-1}[F]$. Since $f$ is a continuous function, we have

$$
Z \text { is a closed subset of } \mathbb{S} \text {. }
$$

From [9, Ch. 2, Pr. 337] it follows that $f\left\lceil Z: Z_{\mathbb{S}} \rightarrow\langle F, \tau\lceil F\rangle\right.$ is a continuous open surjection. Since $\langle F, \tau\lceil F\rangle$ has no isolated points and $f\lceil Z$ is an open map, we see that

$Z$ has no isolated points.

From (35), (36) and [8, (iii) of Theorem 4.6] it follows that $Z_{\mathbb{S}} \cong \mathbb{S}$. And so $\langle F, \tau \mid F\rangle$ is a continuous open image of the Sorgenfrey line.

Corollary 4. Suppose that the Sorgenfrey line is embeddable in a Hausdorff compact space $\langle X, \tau\rangle$. Then $\langle X, \tau\rangle$ is not a continuous open image of the Sorgenfrey line.

Proof. Let $A \subseteq X$ be such that $\left\langle A, \tau\lceil A\rangle \cong \mathbb{S}\right.$. Let $S:=\mathrm{Cl}_{\langle X, \tau\rangle}(A)$, then $\langle S, \tau \mid S\rangle$ is a compactification of $\mathbb{S}$. So from Corollary 3 it follows that

(37) $\langle S, \tau \mid S\rangle$ is not a continuous open image of the Sorgenfrey line.

From Lemma 6 and (37) it follows that $\langle X, \tau\rangle$ is not a continuous open image of the Sorgenfrey line. 


\section{REFERENCES}

[1] A. S. Kechris, Classical Descriptive Set Theory, Graduate Texts in Mathematics, 156 New York: Springer-Verlag, 1995.

[2] K. P. Hart, J. Nagata, and J. E. Vaughan, eds., Encyclopedia of general topology, Elsevier, Amsterdam, 2004.

[3] M. Patrakeev, Metrizable images of the Sorgenfrey line, Topology Proc. 45 (2015), 253-269.

[4] K. Kunen, Set Theory: An Introduction to Independence Proofs, Studies in Logic and the Foundations of Mathematics, 102 Amsterdam-New York: North-Holland Publishing Co., 1980.

[5] N. V. Velichko, Maps of the Sorgenfrey line, Trudy Instituta Matematiki i Mekhaniki UrO RAN 16 2010, no. 1, 52-56 (in Russian); English translation in: Proceedings of the Steklov Institute of Mathematics 272 (2011), suppl. 1, pp. S287-S291.

[6] M. A. Patrakeev, Continuous maps from the Sorgenfrey line onto the Real line, Tomsk State University Journal of Mathematics and Mechanics, 2008, no. 1 (2), 67-75 (in Russian).

[7] S. A. Svetlichny, On projective completeness and projective classes of spaces, Vestnik Moskov. Univ. Ser. 1 Mat. Mekh. 1988, no. 1, 75-77 (in Russian).

[8] D. K. Burke, J. T. Moore, Subspaces of the Sorgenfrey line, Topology Appl. 90 (1-3) (1998) $57-68$.

[9] A. V. Arkhangel'skii and V. I. Ponomarev, Osnovy obschej topologii v zadachakh $i$ uprazhnenijakh, Izdatel'stvo 'Nauka', Moscow, 1974 (in Russian); English translation in: A. V. Arkhangel'skii, V. I. Ponomarev, Fundamentals of general topology: problems and exercises, D. Reidel Publishing Company, 1984.

[10] S. A. Svetlichny, Open mappings of submetrizable spaces, Vestnik Moskov. Univ. Ser. 1 Mat. Mekh. 1988, no. 6, 18-20 (in Russian).

Vlad SMOLin

Krasovski Institute of Mathematics and Mechanics,

Sofia Kovalevskaya Street, 16 , 620990, EKATERINBURG, RUSSiA

E-mail address: SVRusl@yandex.ru 